\title{
Analysis of One Popular Group Signature Scheme
}

\author{
Zhengjun $\mathrm{Cao}^{1,2}$ \\ ${ }^{1}$ Department of Mathematics, Shanghai University, Shanghai, China 200444 \\ ${ }^{2}$ Key Lab of Mathematics Mechanization, Academy of Mathematics and Systems \\ Science, Chinese Academy of Sciences, Beijing, China \\ zjcamss@163.com
}

\begin{abstract}
The group signature scheme [1], ACJT for short, is popular. In this paper we show that it is not secure. It does not satisfy exculpability. The group manager can sign on behalf of any group member. The drawback found in the scheme shows that some inductions are not sound, though they are prevalent in some so-called security proofs.
\end{abstract}

Keywords: group signature, exculpability, anonymity.

\section{Introduction}

Group signatures, introduced by Chaum and Heyst [2], allow individual members to make signatures on behalf of the group. Generally, a group signature must satisfy the following properties [1]:

Unforgeability: Only group members are able to sign messages on behalf of the group.

Anonymity: Given a valid signature of some message, identifying the actual signer is computationally hard for everyone but the group manager.

Unlinkability: Deciding whether two different valid signatures were produced by the same group member is computationally hard.

Traceability: The group manager is always able to open a valid signature and identify the actual signer.

Coalition-resistance: A colluding subset of group members (even if comprised of the entire group) cannot generate a valid signature that the group manager cannot link to one of the colluding group members.

Exculpability: Neither a group member nor the group manager can sign on behalf of other group member.

Group signatures can be used to constitute a very useful primitive in many settings. It has become a hot problem to research group signatures in recent $[3-7]$.

At Crypto'2000, Ateniese et al. [1] proposed a group signature scheme. The authors claimed that the scheme was practical and provably secure coalitionresistant. Recently, we find it is false. The group manager can sign on behalf of any group member. That is to say, the popular group signature scheme does 
not satisfy exculpability. It's the first time to show that the signature scheme is not secure. The attack developed in the paper is novel and interesting. The drawback found in the popular signature scheme shows that some inductions are not sound, though they are prevalent in so-called security proofs.

The rest of the paper is organized as follows. The next section reviews ACJT group signature scheme. An attack is presented in Section 3. Some conclusion remarks are given in Section 4.

\section{Review}

Let $\epsilon>1, k, \ell_{p}$ be security parameters and let $\lambda_{1}, \lambda_{2}, \gamma_{1}, \gamma_{2}$ denote the lengths satisfying

$$
\lambda_{1}>\epsilon\left(\lambda_{2}+k\right)+2, \quad \lambda_{2}>4 \ell_{p}, \quad \gamma_{1}>\epsilon\left(\gamma_{2}+k\right)+2, \quad \gamma_{2}>\lambda_{1}+2 .
$$

Define the integral ranges

$$
\Lambda=] 2^{\lambda_{1}}-2^{\lambda_{2}}, \quad 2^{\lambda_{1}}+2^{\lambda_{2}}[, \quad \Gamma=] 2^{\gamma_{1}}-2^{\gamma_{2}}, 2^{\gamma_{1}}+2^{\gamma_{2}}[.
$$

Finally, let $\mathcal{H}$ be a collision-resistant hash function $\mathcal{H}:\{0,1\}^{*} \rightarrow\{0,1\}^{k}$.

The initial phase involves the group manager (GM) setting the group public key $\mathcal{Y}$ and his secret key $\mathcal{S}$.

\section{SETUP:}

1. Select random secret $\ell_{p}$-bit primes $p^{\prime}, q^{\prime}$ such that $p=2 p^{\prime}+1$ and $q=2 q^{\prime}+1$ are primes. Set the modulus $n=p q$.

2. Choose random elements $a, a_{0}, g, h \in_{R} Q R(n)$ (of order $p^{\prime} q^{\prime}$ ).

3. Choose a random secret element $x \in_{R} Z_{p^{\prime} q^{\prime}}^{*}$ and set $y=g^{x} \bmod n$.

4. The group public key is : $\mathcal{Y}=\left(n, a, a_{0}, y, g, h\right)$.

5. The corresponding secret key (known only to GM) is: $\mathcal{S}=\left(p^{\prime}, q^{\prime}, x\right)$.

Suppose now that a new user wants to join the group. We assume that communication between the user and the group manager is secure. The selection of per-user parameters is done as follows:

\section{JOIN:}

1. User $P_{i}$ generates a secret exponent $\left.\bar{x}_{i} \in R\right] 0,2^{\lambda_{2}}[$, a random integer $\left.\bar{r} \in_{R}\right] 0, n^{2}\left[\right.$ and sends $C_{1}=g^{\bar{x}_{i}} h^{\bar{r}} \bmod n$ to GM and proves him knowledge of the representation of $C_{1}$ w.r.t. bases $g$ and $h$.

2. GM checks that $C_{1} \in Q R(n)$. If this is the case, GM selects $\alpha_{i}$ and $\left.\beta_{i} \in R\right] 0,2^{\lambda_{2}}\left[\right.$ at random and sends $\left(\alpha_{i}, \beta_{i}\right)$ to $P_{i}$.

3. User $P_{i}$ computes $x_{i}=2^{\lambda_{1}}+\left(\alpha_{i} \bar{x}_{i}+\beta_{i} \bmod 2^{\lambda_{2}}\right)$ and sends GM the value $C_{2}=a^{x_{i}} \bmod n$. The user also proves to GM:

(a) that the discrete $\log$ of $C_{2}$ w.r.t. base $a$ lies in $\Lambda$, and 
(b) knowledge of integers $u, v$, and $\omega$ such that

i. $u$ lies in $]-2^{\lambda_{2}}, 2^{\lambda_{2}}[$,

ii. $u$ equals the discrete $\log$ of $C_{2} / a^{2^{\lambda_{1}}}$ w.r.t. base $a$, and iii. $C_{1}^{\alpha_{i}} g^{\beta_{i}}$ equals $g^{u}\left(g^{2^{\lambda_{2}}}\right)^{v} h^{\omega}$.

(The statements (i-iii) prove that the user's membership secret $x_{i}=$ $\log _{a} C_{2}$ is correctly computed from $C_{1}, \alpha_{i}$, and $\beta_{i}$.)

4. GM checks that $C_{2} \in Q R(n)$. If this is the case and all the above proofs were correct, GM selects a random prime $e_{i} \in_{R} \Gamma$ and computes $A_{i}:=\left(C_{2} a_{0}\right)^{1 / e_{i}} \bmod n$. Finally, GM sends $P_{i}$ the new membership certificate $\left[A_{i}, e_{i}\right]$. (Note that $A_{i}=\left(a^{x_{i}} a_{0}\right)^{1 / e_{i}} \bmod n$.)

5. User $P_{i}$ verifies that $a^{x_{i}} a_{0} \equiv A_{i}^{e_{i}} \bmod n$.

Armed with a membership certificate $\left[A_{i}, e_{i}\right]$, a group member can generate anonymous and unlinkable group signatures on a generic message $m \in\{0,1\}^{*}$ :

\section{SIGN:}

1. Generate a random value $\omega \in_{R}\{0,1\}^{2 \ell_{p}}$ and compute:

$$
T_{1}=A_{i} y^{\omega} \bmod n, \quad T_{2}=g^{\omega} \bmod n, \quad T_{3}=g^{e_{i}} h^{\omega} \bmod n .
$$

2. Randomly choose $r_{1} \in_{R} \pm\{0,1\}^{\epsilon\left(\gamma_{2}+k\right)}, \quad r_{2} \in_{R} \pm\{0,1\}^{\epsilon\left(\lambda_{2}+k\right)}$, $r_{3} \in_{R} \pm\{0,1\}^{\epsilon\left(\gamma_{1}+2 \ell_{p}+k+1\right)}, r_{4} \in_{R} \pm\{0,1\}^{\epsilon\left(2 \ell_{p}+k\right)}$ and compute:

$$
\begin{gathered}
d_{1}=T_{1}^{r_{1}} /\left(a^{r_{2}} y^{r_{3}}\right) \bmod n, \quad d_{2}=T_{2}^{r_{1}} / g^{r_{3}} \bmod n \\
d_{3}=g^{r_{4}} \bmod n, \quad d_{4}=g^{r_{1}} h^{r_{4}} \bmod n \\
c=\mathcal{H}\left(g\|h\| y\left\|a_{0}\right\| a\left\|T_{1}\right\| T_{2}\left\|T_{3}\right\| d_{1}\left\|d_{2}\right\| d_{3}\left\|d_{4}\right\| m\right) \\
s_{1}=r_{1}-c\left(e_{i}-2^{\gamma_{1}}\right), \quad s_{2}=r_{2}-c\left(x_{i}-2^{\lambda_{1}}\right), \\
s_{3}=r_{3}-c e_{i} \omega, \quad s_{4}=r_{4}-c \omega \quad(\text { all in } \mathbf{Z}) .
\end{gathered}
$$

3. Output $\left(c, s_{1}, s_{2}, s_{3}, s_{4}, T_{1}, T_{2}, T_{3}\right)$.

A verifier can check the validity of a signature $\left(c, s_{1}, s_{2}, s_{3}, s_{4}, T_{1}, T_{2}, T_{3}\right)$ on the message $m$ as follows:

\section{VERIFY:}

1. Compute

$$
c^{\prime}=\mathcal{H}\left(g\|h\| y\left\|a_{0}\right\| a\left\|T_{1}\right\| T_{2}\left\|T_{3}\right\| d_{1}^{\prime}\left\|d_{2}^{\prime}\right\| d_{3}^{\prime}\left\|d_{4}^{\prime}\right\| m\right)
$$

where

$$
\begin{gathered}
d_{1}^{\prime}=a_{0}^{c} T_{1}^{s_{1}-c 2^{\gamma_{1}}} /\left(a^{s_{2}-c 2^{\lambda_{1}}} y^{s_{3}}\right) \bmod n, \quad d_{2}^{\prime}=T_{2}^{s_{1}-c 2^{\gamma_{1}}} / g^{s_{3}} \bmod n, \\
d_{3}^{\prime}=T_{2}^{c} g^{s_{4}} \bmod n, \quad d_{4}^{\prime}=T_{3}^{c} g^{s_{1}-c 2^{\gamma_{1}}} h^{s_{4}} \bmod n
\end{gathered}
$$

2. Accept the signature if and only if $c=c^{\prime}$ and

$$
\begin{aligned}
& s_{1} \in \pm\{0,1\}^{\epsilon\left(\gamma_{2}+k\right)+1}, \quad s_{2} \in \pm\{0,1\}^{\epsilon\left(\lambda_{2}+k\right)+1}, \\
& \underline{s_{3} \in \pm\{0,1\}^{\epsilon\left(\gamma_{1}+2 \ell_{p}+k+1\right)+1}}, \quad s_{4} \in \pm\{0,1\}^{\epsilon\left(2 \ell_{p}+k\right)+1} .
\end{aligned}
$$


In case of a dispute, GM executes the following procedure:

OPEN:

1. Check the signature's validity via the VERIFY procedure.

2. Recover $A_{i}$ (and thus the identity of $P_{i}$ ) as $A_{i}=T_{1} / T_{2}^{x} \bmod n$. 3. Prove that $\log _{g} y=\log _{T_{2}}\left(T_{1} / A_{i} \bmod n\right)$.

Remark 1: In the original description [1], we observe that

$$
r_{3} \in_{R} \pm\{0,1\}^{\epsilon\left(\gamma_{1}+2 \ell_{p}+k+1\right)}, \quad s_{3} \in \pm\{0,1\}^{\epsilon\left(\lambda_{1}+2 \ell_{p}+k+1\right)+1}
$$

It's not difficult to find it should be corrected to keep the consistency between $r_{3}$ and $s_{3}$.

\section{Analysis}

In this section, we show that ACJT group signature scheme doesn't satisfy exculpability. More precisely, we find the group manager (GM) can sign on behalf of any member if GM replaces Step 2 in the original SETUP phase with following:

2. Choose random elements $a_{0}, g, h \in_{R} Q R(n)$ (of order $p^{\prime} q^{\prime}$ ) and set $a=a_{0}^{t}(\bmod n)$, where $t \in_{R} \mathbf{Z}_{p^{\prime} q^{\prime}}^{*}$.

Then GM records $\left(a^{x_{i}}, A_{i}, e_{i}\right)$ in the JOIN phase (pointing to the member $P_{i}$ ). Note that no member can prevent GM from setting $a=a_{0}^{t}(\bmod n)$.

Using $\left(t, a^{x_{i}}, A_{i}, e_{i}\right)$ and the secret key $\left(p^{\prime}, q^{\prime}\right)$, GM can sign on behalf of the member $P_{i}$. Given a message $m$, GM proceeds as follows:

1. Choose $\omega \in_{R}\{0,1\}^{2 \ell_{p}}$ and compute:

$$
T_{1}=A_{i} y^{\omega} \bmod n, T_{2}=g^{\omega} \bmod n, T_{3}=h^{\omega} \bmod n .
$$

2. Choose $b_{1}, b_{2} \in_{R} \mathbf{Z}_{n}, r_{4} \in_{R} \pm\{0,1\}^{\epsilon\left(2 \ell_{p}+k\right)}$ and compute

$$
\begin{aligned}
& d_{1}=\left(a^{x_{i}}\right)^{b_{1}} y^{b_{2}}, \quad d_{2}=g^{b_{2}}, \quad d_{3}=g^{r_{4}}, \quad d_{4}=g^{b_{1} e_{i}} h^{r_{4}}(\bmod n) . \\
& c=\mathcal{H}\left(g\|h\| y\left\|a_{0}\right\| a\left\|T_{1}\right\| T_{2}\left\|T_{3}\right\| d_{1}\left\|d_{2}\right\| d_{3}\left\|d_{4}\right\| m\right)
\end{aligned}
$$

3. Choose $X \in_{R} \Lambda$ and compute

$$
R_{1}=\left(c+b_{1}\right) e_{i}, \quad R_{2}=c X+t^{-1}\left(c+b_{1}\right), \quad R_{3}=\omega R_{1}-b_{2} \quad(\bmod \phi(n))
$$

4. Choose proper $\rho_{1}, \rho_{2}, \rho_{3} \in \mathbf{Z}$ such that

$$
\begin{aligned}
& r_{1}=R_{1}+\rho_{1} \phi(n) \in \pm\{0,1\}^{\epsilon\left(\gamma_{2}+k\right)} \\
& r_{2}=R_{2}+\rho_{2} \phi(n) \in \pm\{0,1\}^{\epsilon\left(\lambda_{2}+k\right)} \\
& r_{3}=R_{3}+\rho_{3} \phi(n) \in \pm\{0,1\}^{\epsilon\left(\gamma_{1}+2 \ell_{p}+k+1\right)}
\end{aligned}
$$


(Since $R_{1}, R_{2}, R_{3} \in \mathbf{Z}_{n}, n=\left(2 p^{\prime}+1\right)\left(2 q^{\prime}+1\right),\left|p^{\prime}\right|=\left|q^{\prime}\right|=\ell_{p}, \epsilon>1, \gamma_{1}>$ $\epsilon\left(\gamma_{2}+k\right)+2, \quad \gamma_{2}>\lambda_{1}+2, \quad \lambda_{1}>\epsilon\left(\lambda_{2}+k\right)+2$ and $\lambda_{2}>4 \ell_{p}$, it's easy to find $\rho_{1}, \rho_{2}, \rho_{3} \in \mathbf{Z}$ satisfying the above restrictions.)

5. Compute

$$
\begin{gathered}
s_{1}=r_{1}-c\left(e_{i}-2^{\gamma_{1}}\right), \quad s_{2}=r_{2}-c\left(X-2^{\lambda_{1}}\right), \\
s_{3}=r_{3}-c e_{i} \omega, \quad s_{4}=r_{4}-c \omega \quad(\text { all in } \mathbf{Z}) .
\end{gathered}
$$

6. Output $\left(c, s_{1}, s_{2}, s_{3}, s_{4}, T_{1}, T_{2}, T_{3}\right)$.

Correctness: For convenience, denote by $\xi_{i}$ the inverse of $e_{i}$ modulo $\phi(n)$, i.e.,

$$
e_{i} \xi_{i}=1 \bmod \phi(n)
$$

Hence, we have

$$
\begin{aligned}
d_{1}^{\prime} & =a_{0}^{c} T_{1}^{s_{1}-c 2^{\gamma_{1}}} /\left(a^{s_{2}-c 2^{\lambda_{1}}} y^{s_{3}}\right)=a_{0}^{c}\left(A_{i} y^{\omega}\right)^{r_{1}-c e_{i}} /\left(a^{r_{2}-c X} y^{r_{3}-c e_{i} \omega}\right) \\
& =a_{0}^{c}\left(\left(a^{x_{i}} a_{0}\right)^{\xi_{i}}\right)^{\left(r_{1}-c e_{i}\right)} y^{\omega r_{1}-r_{3}} / a^{r_{2}-c X} \\
& =\left(a^{x_{i}}\right)^{r_{1} \xi_{i}-c} a_{0}^{c+r_{1} \xi_{i}-c} y^{\omega r_{1}-r_{3}} / a^{r_{2}-c X}=\left(a^{x_{i}}\right)^{r_{1} \xi_{i}-c} a_{0}^{r_{1} \xi_{i}} y^{\omega r_{1}-r_{3}} / a_{0}^{t\left(r_{2}-c X\right)} \\
& =\left(a^{x_{i}}\right)^{r_{1} \xi_{i}-c} a_{0}^{r_{1} \xi_{i}-t\left(r_{2}-c X\right)} y^{\omega r_{1}-r_{3}}=\left(a^{x_{i}}\right)^{R_{1} \xi_{i}-c} a_{0}^{R_{1} \xi_{i}-t\left(R_{2}-c X\right)} y^{\omega R_{1}-R_{3}} \\
& =\left(a^{x_{i}}\right)^{b_{1}} a_{0}^{c+b_{1}-t\left(c+b_{1}\right) t^{-1}} y^{b_{2}}=\left(a^{x_{i}}\right)^{b_{1}} y^{b_{2}}=d_{1} \quad(\bmod n) \\
d_{2}^{\prime} & =T_{2}^{s_{1}-c 2^{\gamma_{1}}} / g^{s_{3}}=\left(g^{\omega}\right)^{r_{1}-c e_{i}} / g^{r_{3}-c e_{i} \omega} \\
& =g^{\omega r_{1}-r_{3}}=g^{\omega R_{1}-R_{3}}=g^{b_{2}}=d_{2} \quad(\bmod n) \\
d_{3}^{\prime} & =T_{2}^{c} g^{s_{4}}=\left(g^{\omega}\right)^{c} g^{r_{4}-\omega c}=g^{r_{4}}=d_{3} \quad(\bmod n) \\
d_{4}^{\prime} & =T_{3}^{c} g^{s_{1}-c 2^{\gamma_{1}}} h^{s_{4}}=\left(h^{\omega}\right)^{c} g^{r_{1}-c e_{i}} h^{r_{4}-c \omega} \\
& =g^{R_{1}-c e_{i}} h^{r_{4}}=g^{b_{1} e_{i}} h^{r_{4}}=d_{4} \quad(\bmod n)
\end{aligned}
$$

Thus $c^{\prime}=c$. It's easy to check that

$$
\begin{gathered}
s_{1} \in \pm\{0,1\}^{\epsilon\left(\gamma_{2}+k\right)+1}, \quad s_{2} \in \pm\{0,1\}^{\epsilon\left(\lambda_{2}+k\right)+1}, \\
s_{3} \in \pm\{0,1\}^{\epsilon\left(\gamma_{1}+2 \ell_{p}+k+1\right)+1}, \quad s_{4} \in \pm\{0,1\}^{\epsilon\left(2 \ell_{p}+k\right)+1} .
\end{gathered}
$$

Clearly, we also have

$$
T_{1} / T_{2}^{x}=A_{i} y^{\omega} /\left(g^{\omega}\right)^{x}=A_{i} \quad \bmod n
$$

Therefore, the scheme is not exculpable.

Remark 2: The authors [1] claimed that 
First note that due to Corollary 2, GM does not get any information about a user's secret $x_{i}$ apart from $a^{x_{i}}$. Thus, the value $x_{i}$ is computationally hidden from GM. Next note that $T_{1}, T_{2}$, and $T_{3}$ are an unconditionally binding commitments to $A_{i}$ and $e_{i}$. One can show that, if the factorization of $n$ would be publicly known, the interactive proof underlying the group signature scheme is a proof of knowledge of the discrete $\log$ of $A_{i}^{e_{i}} / a_{0}$ (provided that $\ell_{p}$ is larger than twice to output length of the hash function / size of the challenges). Hence, not even the group manager can sign on behalf of $P_{i}$ because computing discrete logarithms is assumed to be infeasible.

But by the above attack, GM is not forced to know a user's secret $x_{i}$ even that $T_{1}, T_{2}$, and $T_{3}$ are an unconditionally binding commitments to $A_{i}$ and $e_{i}$. We should stress that the likes of the above induction are not sound, though they are prevalent in some so-called security proofs.

\section{Conclusion}

In this paper we show that ACJT group signature scheme is insecure. The attack introduced in the paper will be helpful for researching group signature schemes in the future. Incidently, the fair E-cash system [8] directly based on ACJT fails. But it seems that the attack does not apply to the extensions of ACJT proposed in [9]. The extension proposed in [10] appears to resist the attack at the cost of the presence of a trusted third party.

\section{Acknowledgements}

I would like to express my sincere gratitude to my supervisor Professor M.L. Liu for many enlightening suggestions. Thanks also go to those anonymous referees who contributed with their expertise to the final version of the paper. This work is supported by National Natural Science Foundation of China (90304012) and Project 973 (2004CB318000).

\section{References}

[1] G. Ateniese, J. Camenisch, M. Joye, and G. Tsudik. A practical and provably secure coalition-resistant group signature scheme. CRYPTO'2000, LNCS 1880, pp.255-270. Springer-Verlag, 2000.

[2] D. Chaum and E. van Heyst. Group signatures. EUROCRYPT'1991, LNCS 547, pp.257-265. Springer-Verlag, 1992.

[3] D. Song. Practical forward-secure group signature schemes. ACM Symposium on Computer and Communication Security, pp.225-234, November 2001.

[4] E. Bresson and J. Stern. Efficient revocation in group signatures. PKC'2001, LNCS 1992, pp.190-206. Springer-Verlag, 2001.

[5] G. Ateniese and B. de Medeiros. Efficient group signatures without trapdoors. ASIACRYPT'2003, LNCS 2894, pp.246-268. Springer-Verlag, 2003. 
[6] Mihir Bellare, Daniele Micciancio, Bogdan Warinschi. Foundations of Group Signatures: Formal Definitions, Simplified Requirements, and a Construction Based on General Assumptions. EUROCRYPT'2003, LNCS 2656, pp.614-629. SpringerVerlag, 2003.

[7] D. Boneh, X. Boyen and H. Shacham. Short group signatures. CRYPTO'2004, LNCS 3152, pp.41-55. Springer-Verlag, 2004.

[8] Greg Maitland and Colin Boyd. Fair electronic cash based on a group signature scheme. Information and Communications Security'2001, LNCS 2229, pp.461465. Springer-Verlag, 2001.

[9] J. Camenisch and J. Groth. Group Signatures: Better Efficiency and New Theoretical Aspects: Forth Conference on Security in Communication Networks-SCN'04. LNCS 3352, pp.120-133. Springer-Verlag, 2005.

[10] G. Tsudik, S. Xu. Accumulating composites and improved group signing. ASIACRYPT 2003, LNCS 2894, pp.269-286. Springer-Verlag, 2003. 